\title{
Role of adjuvant chemotherapy after pneumonectomy for non-small cell lung cancer
}

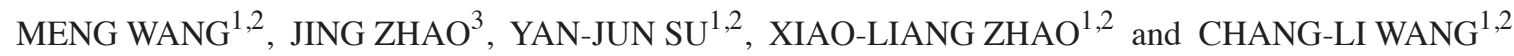 \\ ${ }^{1}$ Department of Lung Cancer, Cancer Institute and Hospital, Tianjin Medical University, Key Laboratory of \\ Cancer Prevention and Therapy Tianjin; ${ }^{2}$ Tianjin Diagnosis and Treatment Center of Lung Cancer; \\ ${ }^{3}$ Department of Breast Cancer, Cancer Institute and Hospital, Tianjin Medical University, Tianjin 300060, P.R. China
}

Received April 23, 2012; Accepted July 20, 2012

DOI: $10.3892 / \mathrm{ol} .2012 .892$

\begin{abstract}
Adjuvant chemotherapy is used as an alternative treatment for non-small cell lung cancer (NSCLC); however, the efficiency of post-pneumonectomy adjuvant chemotherapy in NSCLC has not been clarified. In the present study, patients who benefited from adjuvant chemotherapy with TP/NP/GP were identified. A total of 217 patients who underwent pneumonectomy were identified in this study. Of these, 87 underwent pneumonectomy combined with adjuvant chemotherapy (TP/NP/GP regimen) and 130 underwent pneumonectomy only in the initial management. The primary endpoint of the present study was overall survival. Actuarial survival analysis was conducted using the Kaplan-Meier method. Postoperative adjuvant chemotherapy significantly improved the survival rate of patients who underwent left pneumonectomy and in patients with a preoperative forced expiratory volume in $1 \mathrm{sec}$ (FEV1) greater than or equal to 2 1. Age had no effect on the survival rate of patients with or without postoperative adjuvant therapy. Post-pneumonectomy adjuvant chemotherapy is an efficient therapy in NSCLC for patients with preoperative FEV1 greater than or equal to 21 or who received left pneumonectomy.
\end{abstract}

\section{Introduction}

Pneumonectomy is a standard surgical approach for central lung cancer patients. Extensive pulmonary resection offers curative treatment in a large population of patients and has been widely accepted. Postoperative adjuvant chemotherapy in non-small cell lung cancer (NSCLC) patients has been reported to be an efficient therapy, resulting in significant improvement in overall survival (OS) (1-4). Previous studies have focused

Correspondence to: Dr Chang-Li Wang, Department of Lung Cancer, Cancer Institute and Hospital, Tianjin Medical University, He Xi Qu Huan Hu Xi Road, Tianjin 300060, P.R. China E-mail: wm_1975@live.cn

Key words: non-small cell lung cancer, pneumonectomy, adjuvant chemotherapy, forced expiratory volume in 1 second on postoperative adjuvant chemotherapy, including mixed exploratory thoracotomy, wedge resections, lobectomy and pneumonectomy with or without extended resection of the chest wall (1-4). However, no study focusing solely on pneumonectomy has been published. Moreover, in one study, not all patients were treated with third generation chemotherapy agents such as taxol, vinorelbine and gemcitabine. As no studies on the effect of adjuvant third generation chemotherapy agents for NSCLC after only pneumonectomy have been performed, the role of post-pneumonectomy adjuvant chemotherapy is unclear and more data are required.

Long-term respiratory complications have been considered to be the most severe events after pneumonectomy, since 'one-lung' patients are at a high risk of respiratory failure. Chemotherapy is an additional risk factor for respiratory complications $(5,6)$. Therefore, the administration of adjuvant chemotherpy after pneumonectomy for NSCLC is controversial.

In the present study, we investigated the effect of adjuvant chemotherapy on NSCLC after pneumonectomy and identified a population of patients who benefited from adjuvant chemotherapy with TP/NP/GP. Our data may aid the identification of patients who are likely to benefit from post-pneumonectomy adjuvant chemotherapy in NSCLC. This is the first intensive observation of the effect of adjuvant chemotherapy (third generation) combined with pneumonectomy.

\section{Patients and methods}

Patients. A retrospective chart review analysis was performed on NSCLC patients who underwent pneumonectomy. A total of 261 NSCLC patients were treated with pneumonectomy at the Tianjin Cancer Hospital of Tianjin Medical University (between January 2000 and January 2007). A total of 6 patients $(2.3 \%)$ who underwent pneumonectomy succumbed within 30 days of surgery and 3 patients $(1.1 \%)$ underwent incomplete resection. Patients who had received radiation therapy, biotherapy or induction therapy (stage $\mathrm{pN} 2$ ), who were lost to follow-up or succumbed to postoperative complications and patients with an ECOG performance status of 2-3 who did not recover within 6 weeks of surgery were excluded from the study. The remaining 217 patients who underwent pneumonectomy and had an ECOG performance status of 0-1 6 weeks 
after surgery were identified for this study. Among these patients, 130 underwent pneumonectomy as the only therapy in the initial treatment of their disease; 79 patients underwent pneumonectomy and 3 or 4 cycles of adjuvant chemotherapy of TP/NP/GP regimen [TP: taxol, $135-175 \mathrm{mg} / \mathrm{m}^{2}$, day 1 and carboplatin, area under the ROC curve $(A U C)=5$, day 1 ; NP: Navelbine, $25 \mathrm{mg} / \mathrm{m}^{2}$, days 1 and 8 and carboplatin, $\mathrm{AUC}=5$, day 1; GP: gemcitabine, $1250 \mathrm{mg} / \mathrm{m}^{2}$, days 1 and 8 and carboplatin, $\mathrm{AUC}=5$, day 1]. A total of 8 patients who were treated with only 1 or 2 cycles of chemotherapy due to toxicity were also included into this study. These two groups of patients had similar clinical stages of disease and preoperative respiratory function. Blood count $\left(\mathrm{WBC}>3.0 \times 10^{9} \mathrm{1}^{-1}\right.$; absolute neutrophil count $>1500 \times 10^{9} \mathrm{l}^{-1}$; platelets $>100 \times 10^{9} \mathrm{l}^{-1}$ ), liver function (aspartate aminotransferase/alanine aminotransferase and alkaline phosphatase $\leq 2$ times the upper limit of normal was accepted) and renal function (serum creatinine $\leq 2 \mathrm{mg} / \mathrm{dl}$ via biochemistry tests) were monitored in all patients prior to adjuvant chemotherapy.

Informed consent was obtained either from the patient or the patient's family. The study was approved by the ethics committee of Tianjin Medical University, Tianjin, China.

Diagnosis. All patients underwent preoperative computed tomography (CT) scans of the thorax and upper abdomen or ultrasound of the upper abdomen, fiber-optic bronchoscopy with cytological specimens, bone scintigraphy and brain CT. Preoperative respiratory function was assessed routinely by blood gas analysis, spirometry and lung perfusion scan.

Standard pneumonectomy was defined as the intrapericardial or extrapericardial removal of the entire lung, associated with radical mediastinal lymph node dissection without any resection of mediastinal, chest wall or diaphragmatic structure.

If there were any signs of metastasis to the mediastinum (lymph nodes $>1.5 \mathrm{~cm}$ ), mediastinoscopy was performed as routine. If the procedure yielded negative results, the patients underwent thoracotomy. If the procedure had positive results, the patients underwent induction therapy (excluded from the study). However, certain patients were diagnosed as having pathological N2 disease following surgery. No patients had a positron emission tomography scan due to the unavailability of this equipment in our city prior to 2007. Pathological and clinical TNM staging was determined according to the guidelines of the American Joint Committee on Cancer $(7,8)$. Histological subtypes included squamous cell, adenocarcinoma and other types, such as bronchoalveolar carcinoma, large cell carcinoma and adenosquamous.

Evaluation. Preoperative patient variables were collected in the surgical database. Survival time was calculated from the date of pathological diagnosis of lung cancer. OS was used as the primary endpoint of the study. OS was compared between the adjuvant chemotherapy and pneumonectomy only groups, taking into account age, preoperative respiratory function and site of surgery.

Follow-up. Follow-up data were collected directly from our outpatient clinic records. All patients followed a standardized program of clinical and instrumental examinations. The median follow-up time was 35 months (range, 3-125).
Table I. Demographics.

\begin{tabular}{lccc}
\hline & $\begin{array}{c}\text { Adjuvant } \\
\text { chemotherapy } \\
\text { Variable }\end{array}$ & $\begin{array}{c}\text { Pneumonectomy } \\
\text { only } \\
\mathrm{n}=130\end{array}$ & P-value \\
\hline Male & 68 & 98 & 0.64 \\
Age $\geq 65$ years & 34 & 35 & 0.06 \\
P-stage & & & 0.09 \\
IB & 12 & 29 & \\
II & 30 & 52 & \\
IIIA & 45 & 49 & \\
FEV1 (1), mean \pm SD & $1.95 \pm 0.43$ & $1.88 \pm 0.38$ & 0.21 \\
\hline
\end{tabular}

FEV1, forced expiratory volume in $1 \mathrm{sec}$.

Statistical analysis. Actuarial survival analysis was calculated using the Kaplan-Meier method. The log-rank test was used to compare the OS between the groups. $\mathrm{P}<0.05$ was considered to indicate a statistically significant result All statistical analyses were performed using SPSS software (version 13.0 for Windows).

\section{Results}

Patient population. Demographic data of the adjuvant chemotherapy and pneumonectomy only groups are shown in Table I. The mean age of patients involved in this study was 56 years (range, 32-75). The number of patients treated with left pneumonectomy was 173 . The number of patients with preoperative forced expiratory volume in 1 sec (FEV1) $\geq 21$ was 86 . The tumor pathology stages included stage IB $(13.8 \%)$, stage II $(34.5 \%)$ and stage IIIA $(51.7 \%)$ in the adjuvant chemotherapy group and stage IB (22.3\%), stage II (40\%) and stage IIIA $(37.7 \%)$ in the pneumonectomy only group. The histological type of the tumors was squamous cell (77\%), adenocarcinoma (17\%) and other (6\%) in the adjuvant chemotherapy group and squamous cell $(72 \%)$, adenocarcinoma (23\%) and other (5\%) in the pneumonectomy only group. In summary, the demographic data were similar in the two groups with regard to age, gender, histological type of tumors, tumor P-stage, ECOG performance status and preoperative respiratory function.

Mortality and complications. During the follow-up period, 217 cases were analyzed and 173 patients succumbed. Of the 173 patients, $89 \%$ succumbed to cancer and $11 \%$ succumbed to other causes. Of the 6 patients in the surgery group who succumbed to other causes, 4 succumbed to respiratory failure, 1 to myocardial infarction and 1 to unknown causes. Of the 13 patients in the chemotherapy group who succumbed to other causes, 1 succumbed to myocardial infarction and 12 to respiratory failure (6 from the right pneumonectomy group, 6 from the left pneumonectomy group). Complications of post-pneumonectomy adjuvant chemotherapy included bronchopleural fistula ( 2 cases), pneumonia (11 cases) and interstitial lung disease (4 cases). No unusual associated 
Table II. Cox proportional hazard ratio model showed that adjuvant chemotherapy was an independent prognostic factor in patients with preoperative FEV1 $\geq 21$.

\begin{tabular}{lccc}
\hline Variable & $\begin{array}{c}\text { Hazard } \\
\text { ratio }\end{array}$ & $\begin{array}{c}95 \% \text { confidence } \\
\text { interval }\end{array}$ & P-value \\
\hline $\begin{array}{l}\text { Adjuvant } \\
\text { chemotherapy (yes/no) }\end{array}$ & 0.49 & $0.28-0.86$ & 0.012 \\
P-stage (IB/II, IIIA) & 0.33 & $0.15-0.70$ & 0.004 \\
$\begin{array}{l}\text { Age, years (<65/ } \geq 65) \\
\text { Gender (male/female) }\end{array}$ & & & 0.265 \\
$\begin{array}{l}\text { Pneumonectomy } \\
\text { (left/right) }\end{array}$ & & & 0.776 \\
\hline
\end{tabular}

FEV1, forced expiratory volume in 1 sec.

A

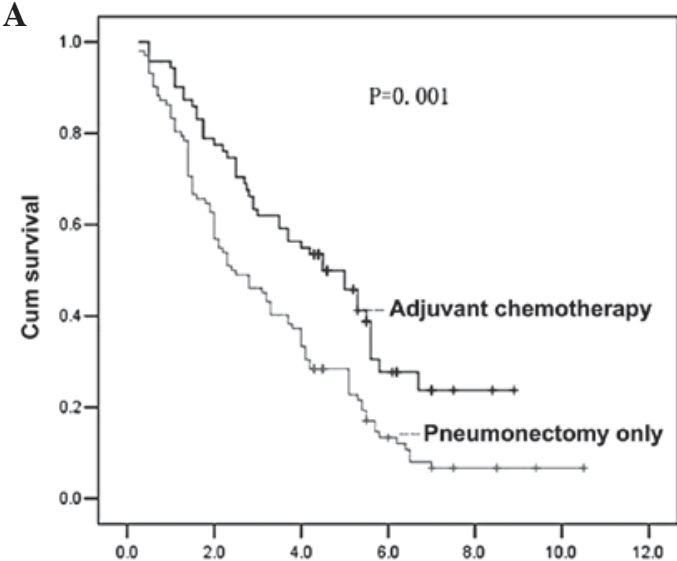

B

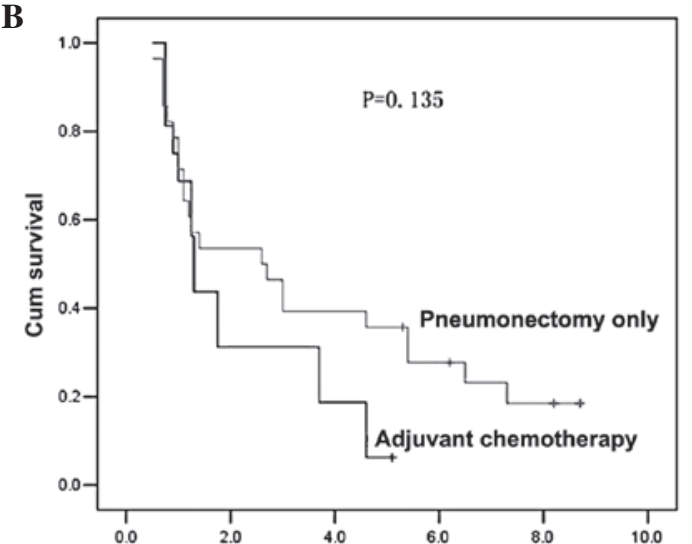

Figure 1. Site of pneumonectomy affects the outcome of adjuvant chemotherapy. Kaplan-Meier actuarial overall survival was calculated from the time of pathological diagnosis. (A) Overall survival of patients who underwent left pneumonectomy in the adjuvant chemotherapy and pneumonectomy only groups. (B) Overall survival of patients who underwent right pneumonectomy in the adjuvant chemotherapy and pneumonectomy only groups.

toxicity was recorded during the chemotherapy. No patients succumbed to treatment-related toxicity during chemotherapy.

Survival analysis. The 5-year actuarial OS rate of the patients was $32.1 \%$ in the present study. The 5-year actuarial OS rates of the left and right pneumonectomy groups were 34.3 and
Table III. Cox proportional hazard ratio model showed that adjuvant chemotherapy was an independent prognostic factor in patients who underwent left pneumonectomy.

\begin{tabular}{lccc}
\hline Variable & $\begin{array}{c}\text { Hazard } \\
\text { ratio }\end{array}$ & $\begin{array}{c}95 \% \text { confidence } \\
\text { interval }\end{array}$ & P-value \\
\hline $\begin{array}{l}\text { Adjuvant } \\
\text { chemotherapy (yes/no) }\end{array}$ & 0.61 & $0.42-0.88$ & 0.009 \\
P-stage (IB/II, IIIA) & 0.34 & $0.21-0.57$ & 0.000 \\
Age, years $(<65 / \geq 65)$ & & & 0.599 \\
Gender $($ male/female) & & & 0.999 \\
FEV1, $1(<2 / \geq 2)$ & & & 0.236
\end{tabular}

FEV1, forced expiratory volume in $1 \mathrm{sec}$.

A

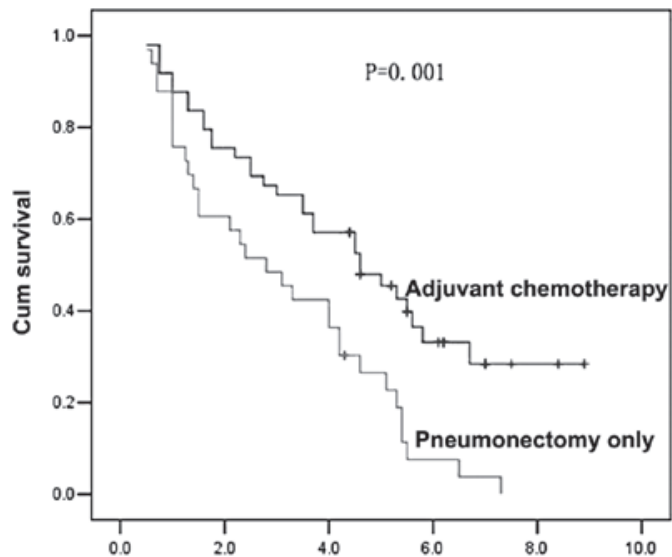

B

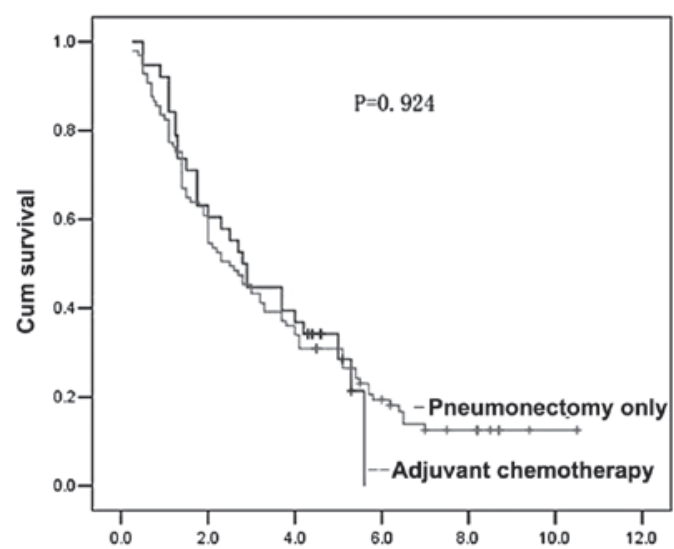

Figure 2. Preoperative respiratory function affects the outcome of adjuvant chemotherapy. (A) Overall survival of patients with preoperative FEV1 $\geq 21$ in the adjuvant chemotherapy and pneumonectomy only groups. (B) Overall survival of patients with preoperative FEV1 $<21$ in the adjuvant chemotherapy and pneumonectomy only groups. FEV1, forced expiratory volume in $1 \mathrm{sec}$.

$26.2 \%$, respectively $(\mathrm{P}=0.35)$. The 5-year actuarial OS rates of the adjuvant chemotherapy and pneumonectomy only groups were 36.2 and $30.1 \%$, respectively $(\mathrm{P}=0.067)$.

The univariate analysis of OS demonstrated that postoperative adjuvant chemotherapy significantly increased the survival rate in patients who underwent left pneumonectomy 
$(\mathrm{P}=0.001$; Fig. 1A) and in patients with preoperative FEV1 $\geq 21$ ( $\mathrm{P}=0.001$; Fig. 2A).

The univariate analysis of OS demonstrated that postoperative adjuvant chemotherapy did not significantly increase the survival rate in patients with right pneumonectomy (Fig. 1B), preoperative FEV1 $<21$ (Fig. $2 \mathrm{~B})$, age $\geq 65$ years $(\mathrm{P}=0.105)$ and age $<65$ years $(\mathrm{P}=0.091$; data not shown).

To clarify our analysis, multivariate analysis was performed. The Cox proportional hazards ratio model revealed that adjuvant chemotherapy was an independent prognostic factor for better survival in patients with preoperative FEV1 $\geq 21$ and patients who underwent left pneumonectomy (Tables II and III).

\section{Discussion}

The present study is the first to evaluate the effect of adjuvant third generation chemotherapy agents on NSCLC after only pneumonectomy and analyze the population of patients who are likely to benefit from adjuvant chemotherapy. We have demonstrated that a low respiratory capacity, assessed as FEV1, appears to be the most important predictor of a high risk of complications following lung resection (9). The British Thoracic Society (BTS) guidelines state that no further respiratory function tests are required for a pneumonectomy if the post-bronchodilator FEV1 is above 2.01 , provided that there is no evidence of interstitial lung disease or unexpected disability due to shortness of breath (10). The preoperative FEV1 is likely to be the most important marker of respiratory functions.

Postoperative cardiopulmonary function and exercise capacity have been examined following pulmonary resection and demonstrated to be persistently decreased only after pneumonectomy $(11,12)$. The impairment of respiratory function after a pneumonectomy may be explained by overdistention and emphysematous changes of the contralateral lung (which may show evidence of histological hyperplasia and loss of elasticity), an increase in the airway resistance resulting in alveolar gas mixing, arterial desaturation or late pulmonary failure. Consistent with this point, it has been reported that the impairment of the alveolocapillary membrane is more likely to occur after chemotherapy (13) which may be synergic with other risk factors, including age or fluid overload causing lung damage (14). Therefore, chemotherapy after pneumonectomy may aggravate lung damage. Our data show that postoperative adjuvant chemotherapy significantly improves the survival rate of patients with preoperative FEV1 greater than or equal to 21 , but has no effect on patients with preoperative FEV1 of less than 21 . Preoperative respiratory functions affect the results of chemotherapy following pneumonectomy. The better respiratory function of the NSCLC patients before pneumonectomy, the better the effect of postoperative adjuvant chemotherapy. This is most likely due to patients with good respiratory functions rarely succumbing to respiratory failure caused by postoperative adjuvant chemotherapy. Winton et al (15) reported that adjuvant treatment with third generation chemotherapy agents may be safely administered in the outpatient setting with limited toxicity and is beneficial for NSCLC patients; $25 \%$ of the patients accepted pneumonectomy. This report is in line with our results. In summary, chemotherapy in
NSCLC patients with good performance status after complete resection is beneficial.

It is well known that the impairment of respiratory function after right pneumonectomy is more serious than that following left pneumonectomy. Martin et al reported that pneumonectomy on the right side is associated with a mortality rate of $24 \%$ at 3 months, whereas there was no mortality following left pneumonectomy (16). The safety of pneumonectomy, especially right pneumonectomy after induction therapy, has been questioned in several studies $(16,17)$. In the present study, $37 \%$ $(6 / 16)$ of the patients with right pneumonectomy succumbed to respiratory failure in the adjuvant chemotherapy group, whereas only $8 \%(6 / 71)$ of patients with left pneumonectomy succumbed to respiratory failure in the adjuvant chemotherapy group. Respiratory failure was one of the main causes of mortality in patients who underwent right pneumonectomy following adjuvant chemotherapy in the present study. Our data show that postoperative adjuvant chemotherapy significantly improved the survival rate of patients who underwent left, but not right, pneumonectomy.

Since the impairment of respiratory function after right pneumonectomy is more serious than that following left pneumonectomy and the mortality rate of right pneumonectomy is high, between January 2000 and January 2007, a total of 44 NSCLC patients treated with right pneumonectomy were identified in our hospital. As the number is low, it is possible to generate statistical errors. This is a preliminary study, further study will continue in the future with an increasing number of patients who underwent right pneumonectomy.

In the present study, we investigated the correlation between age and the effect of chemotherapy following pneumonectomy. We found that there was no significant difference between the survival rate in patients with or without postoperative adjuvant chemotherapy $(\mathrm{P}>0.05)$ in patients aged 65 years or over or those younger than 65 . In conclusion, the age of the patients does not affect the result of chemotherapy following pneumonectomy.

This is a retrospective study, which allows the analysis of the outcomes of a large number of patients treated in a relatively uniform manner. The results of our study suggest that adjuvant chemotherapy following pneumonectomy benefits patients with preoperative FEV1 of 21 or above or patients who undergo left pneumonectomy. The age of patients is not a significant factor.

\section{Acknowledgements}

We would like to thank Dr Hong Yang for assistance in editing this manuscript. We also thank the Division of Medical Records, Cancer Hospital of Tianjin Medical University for technical assistance with the project.

\section{References}

1. Niiranen A, Niitamo-Korhonen S, Kouri M, Assendelft A, Mattson K and Pyrhönen S: Adjuvant chemotherapy after radical surgery for non-small-cell lung cancer: a randomized study. J Clin Oncol 10: 1927-1932, 1992.

2. Wada H, Hitomi S and Teramatsu T: Adjuvant chemotherapy after complete resection in non-small-cell lung cancer. West Japan Study Group for Lung Cancer Surgery. J Clin Oncol 14: 1048-1054, 1996 
3. Kato H, Tsuboi M, Ohta M, Hata E, Tsubota N, Hamajima N, et al: A randomized phase III trial of adjuvant chemotherapy with UFT for completely resected pathological stage I (T1N0M0, T2NOM0) adenocarcinoma of the lung. Proc Am Soc Clin Oncol 22: abstract $2498,2003$.

4. Arriagada R, Bergman B, Dunant A, Le Chevalier T, Pignon JP and Vansteenkiste J; International Adjuvant Lung Cancer Trial Collaborative Group: Cisplatin-based adjuvant chemotherapy in patients with completely resected non-small-cell lung cancer. $\mathrm{N}$ Engl J Med 350: 351-360, 2004.

5. Roberts JR, Eustis C, Devore R, Carbone D, Choy H and Johnson D: Induction chemotherapy increases perioperative complications in patients undergoing resection for non-small cell lung cancer. Ann Thorac Surg 72: 885-888, 2001.

6. Novoa N, Varela G and Jimenez MF: Morbidity after surgery for non-small cell lung carcinoma is not related to neoadjuvant chemotherapy. Eur J Cardiothorac Surg 20: 700-704, 2001.

7. Mountain CF: Value of the new TNM staging system for lung cancer. Chest 96 (1 Suppl): 47S-49S, 1989.

8. Mountain CF: Revisions in the International System for Staging Lung Cancer. Chest 111: 1710-1717, 1997.

9. Wang J, Olak J, Ultmann RE and Ferguson MK: Assessment of pulmonary complications after lung resection. Ann Thorac Surg 67: 1444-1447, 1999

10. British Thoracic Society; Society of Cardiothoracic Surgeons of Great Britain and Ireland Working Party: BTS guidelines: guidelines on the selection of patients with lung cancer for surgery. Thorax 56: 89-108, 2001.

11. Nugent AM, Steele IC, Carragher AM, McManus K, McGuigan JA, Gibbons JR, et al: Effect of thoracotomy and lung resection on exercise capacity in patients with lung cancer. Thorax 54: 334-338, 1999.
12. Larsen KR, Svendsen UG, Milman N, Brenøe J and Petersen B: Cardiopulmonary function at rest and during exercise after resection for bronchial carcinoma. Ann Thorac Surg 64: 960-964, 1997.

13. Stover DE and Kaner RJ: Adverse effects of treatment: pulmonary toxicity. In: Cancer Principles \& Practice of Oncology. De Vita VT, Hellman S and Rosenberg AA (eds). 7th edition. Lippincott Williams \& Willkins, Philadelphia, pp2539-2545, 2004.

14. Chap L, Shpiner R, Levine M, Norton L, Lill M and Glaspy J: Pulmonary toxicity of high-dose chemotherapy for breast cancer: a non-invasive approach to diagnosis and treatment. Bone Marrow Transplant 20: 1063-1067, 1997.

15. Winton T, Livingston R, Johnson D, Rigas J, Johnston M, Butts C, et al; National Cancer Institute of Canada Clinical Trials Group; National Cancer Institute of the United States Intergroup JBR.10 Trial Investigators: Vinorelbine plus cisplatin vs. observation in resected non-small-cell lung cancer. N Engl J Med 352: 2589-2597, 2005.

16. Martin J, Ginsberg RJ, Abolhoda A, Bains MS, Downey RJ, Korst RJ, et al: Morbidity and mortality after neoadjuvant therapy for lung cancer: the risks of right pneumonectomy. Ann Thorac Surg 72: 1149-1154, 2001.

17. Doddoli C, Barlesi F, Trousse D, Robitail S, Yena S, Astoul P, et al: One hundred consecutive pneumonectomies after induction therapy for non-small cell lung cancer: an uncertain balance between risks and benefits. J Thorac Cardiovasc Surg 130: 416-425, 2005 